\title{
Production and composition of group B streptococcal membrane vesicles varies across diverse lineages
}

\author{
Cole R. McCutcheon ${ }^{1}$, Jennifer A. Gaddy ${ }^{2,3,4}$, David M. Aronoff ${ }^{2,3,5}$, \\ Margaret G. Petroff ${ }^{1,6^{*}}$, and Shannon D. Manning ${ }^{1^{*}}$
}

${ }^{1}$ Department of Microbiology and Molecular Genetics, Michigan State University, East Lansing, MI, 48823

${ }^{2}$ Department of Medicine, Division of Infectious Disease, Vanderbilt University Medical Center, Nashville, TN

${ }^{3}$ Department of Pathology, Microbiology, and Immunology, Vanderbilt University Medical Center, Nashville, TN

${ }^{4}$ Tennessee Valley Healthcare System, Department of Veterans Affairs, Nashville, TN

${ }^{5}$ Department of Obstetrics and Gynecology, Vanderbilt University Medical Center, Nashville, $\mathrm{TN}$

${ }^{6}$ Department of Pathobiology and Diagnostic Investigation, Michigan State University, East Lansing, MI, 48823

*Co-corresponding authors: mannin71@msu.edu; petrof10@msu.edu 


\section{ABSTRACT}

3 Although the neonatal and fetal pathogen Group B Streptococcus (GBS) asymptomatically

4 colonizes the vaginal tract of $\sim 30 \%$ of pregnant women, only a fraction of their offspring

5 develops invasive disease. We and others have postulated that these dimorphic clinical

6 phenotypes are driven by strain variability; however, the bacterial factors that promote these

7 divergent clinical phenotypes remain unclear. It was previously shown that GBS produces

8 membrane vesicles (MVs) that contain active virulence factors capable of inducing adverse

9 pregnancy outcomes. Because the relationship between strain variation and vesicle composition

10 or production is unknown, we sought to quantify MV production and examine the protein

11 composition, using label-free proteomics on MVs produced by diverse clinical GBS strains

12 representing three phylogenetically distinct lineages. We found that MV production varied across

13 strains, with certain strains displaying nearly two-fold increases in production relative to others.

14 Hierarchical clustering and principal component analysis of the proteomes revealed that MV

15 composition is lineage-dependent but independent of clinical phenotype. Multiple proteins that

16 contribute to virulence or immunomodulation, including hyaluronidase, C5a peptidase, and

17 sialidases, were differentially abundant in MVs, and were partially responsible for this

18 divergence. Together, these data indicate that production and composition of GBS MVs vary in a

19 strain-dependent manner, suggesting that MVs have lineage-specific functions relating to

20 virulence. Such differences may contribute to variation in clinical phenotypes observed among

21 individuals infected with GBS strains representing distinct lineages. 


\section{Introduction}

Group B Streptococcus (GBS) is an opportunistic pathogen that asymptomatically

26 colonizes $\sim 30 \%$ of women either vaginally or rectally (1). In individuals with a compromised or

27 altered immune state, including pregnant women, neonates, the elderly, and people living with

28 diabetes mellitus, GBS can cause severe infections (1). Presentation of disease is variable

29 between individuals: in elderly patients and neonates, GBS infection typically presents as

30 septicemia, whereas in pregnant women it more commonly causes chorioamnionitis, preterm

31 birth, or stillbirth $(2,3)$.

32 Despite the high prevalence of GBS colonization during pregnancy, only a fraction of

33 babies born to colonized mothers develop an infection. In the United States pregnant individuals

34 colonized with GBS are given antibiotics to reduce the risk of neonatal GBS infection, but even

35 without such prophylaxis most neonates born to GBS-colonized mothers remain infection-free

36 (4). The factors that determine whether a neonate develops GBS sepsis or not are incompletely

37 understood, but evidence implicates bacterial strain variation as a key factor. For example,

38 certain polysaccharide capsular serotypes of GBS are much more common at causing perinatal

39 infections than others (5).

Outside of capsular serotyping, the application of multilocus sequence typing (MLST)

41 has demonstrated that GBS isolates comprise multiple sequence types (STs) that are

42 differentially correlated with disease outcomes (6). While ST-12 strains have been associated

43 with asymptomatic colonization (7), ST-1 and ST-17 strains have been linked to invasive disease

44 in adults and neonates, respectively $(6,8,9)$. Moreover, our group has previously shown that

45 different STs interact variably with host cells. ST-17 strains, for instance, had an enhanced

46 ability to attach to gestational tissues, elicited stronger proinflammatory responses, and could 
47 persist longer inside macrophages than other STs (10-12). Conversely, ST-12 strains were found

48 to display increased tolerance to ampicillin relative to ST-17 strains (12), highlighting the

49 divergence of these lineages and variation in the ability to withstand different stressors. The

50 mechanisms underlying these strain-dependent differences, however, are poorly understood.

$51 \quad$ Many bacteria produce membrane vesicles (MVs) of varying sizes (20-500 nm)

52 containing toxins and other virulence factors that can modulate immune responses and influence

53 pathogenesis (13). In addition, GBS can produce MVs that have been implicated in driving

54 infection risk, though this remains an area in need of more research $(14,15)$. While the exact role

55 of GBS MVs in pathogenesis is not clear, intra-amniotic injection of GBS MVs produced by an

56 invasive ST-7 strain induced preterm birth and intrauterine fetal death in mice (14). GBS MVs

57 were also found to contain active virulence factors that could weaken murine gestational

58 membranes, stimulate immune cell recruitment, and lyse host cells $(14,15)$. Hence, an important,

59 unanswered question is whether MVs derived from strains belonging to distinct phylogenetic

60 lineages and clinical sources vary in composition and pathogenic potential.

In this study, we sought to compare the quantity and protein composition of MVs

62 produced by genetically distinct GBS strains and evaluate the relationships between proteomic

63 profiles, strain characteristics, and clinical presentation. To accomplish these goals, we isolated

$64 \mathrm{MVs}$ from six clinical strains representing three phylogenetic lineages (ST-1, ST-12, and ST-17),

65 and used label-free proteomics to define the protein composition. Using this approach, we report

66 that MV production and composition varies in a strain and ST-dependent manner, highlighting

67 the importance of strain diversity on pathogenic potential. 


\section{Bacterial strains}

74 as described $(16,17)$; the strain names have been abbreviated for clarity. The invasive isolates

75 GB37, GB411, and GB1455 were isolated from the blood or cerebrospinal fluid of infants with

76 early onset GBS disease (16), while the colonizing isolates GB20, GB112, and GB653 were

77 isolated from vaginal/rectal swabs from asymptomatically colonized mothers before or after

78 childbirth (17). These isolates were previously characterized by MLST and capsular (cps)

79 serotyping $(7,9)$ and represent the following three common ST, serotype combinations: ST-1,

80 cpsV (GB20, GB37), ST-12, cpsII (GB653, GB1455), and ST-17, cpsIII (GB112, GB411).

81 Strains were cultured using Todd-Hewitt Broth (THB) or Todd-Hewitt Agar (THA) (BD

82 Diagnostics, Franklin Lakes, New Jersey, USA) overnight at $37^{\circ} \mathrm{C}$ with $5 \% \mathrm{CO}_{2}$.

\section{Membrane vesicle (MV) isolation and purification}

85 The isolation and purification of MVs was performed as described $(14,18-20)$, with some

86 modifications. Briefly, overnight THB cultures were diluted 1:50 into fresh broth and grown to

87 late logarithmic phase (optical density at $600 \mathrm{~nm}, \mathrm{OD}_{600}=0.9$ ). Aliquots of culture were serially

88 diluted and plated on THA for bacterial enumeration. Cultures were centrifuged at $2000 \mathrm{x} \mathrm{g}$ for

8920 minutes at $4^{\circ} \mathrm{C}$. Supernatants were collected and re-centrifuged at $8500 \mathrm{x} \mathrm{g}$ for 15 minutes at

$90 \quad 4^{\circ} \mathrm{C}$, followed by filtration through a $0.22 \mu \mathrm{m}$ filter and concentration using Amicon Ultra-15

91 centrifugal filters (10k Da cutoff) (Millipore Sigma, Burlington, MA, USA). Concentrated

92 supernatants were subjected to ultracentrifugation for 2 hours at $150,000 \mathrm{xg}$ at $4^{\circ} \mathrm{C}$. For 
93 quantification, pellets were washed by resuspending in PBS, re-pelleting at $150,000 \times \mathrm{g}$ at $4^{\circ} \mathrm{C}$,

94 and resuspending in PBS; pellets were stored at $-80^{\circ} \mathrm{C}$ until usage.

95 For proteomics, pellets were resuspended in PBS and purified using qEV Single size

96 exclusion columns (IZON Science, Christchurch, New Zealand) per the manufacturer's

97 instructions. MV fractions were collected and re-concentrated using the Amicon Ultra-4

98 centrifugal filters (10 kDa cutoff) (MilliporeSigma, Burlington, Massachusetts, USA) and

99 brought to a final volume of $100 \mu \mathrm{L}$ in PBS. To preserve the integrity of vesicle proteins,

100 ProBlock Gold Bacterial Protease Inhibitor Cocktail (GoldBio, St. Louis, Missouri, USA) was

101 added. MVs were stored at $-80^{\circ} \mathrm{C}$ until usage.

\section{Electron microscopy}

To visualize GBS MVs, scanning electron microscopy (SEM) was performed on bacterial

105 cultures grown to stationary phase in THB. Culture aliquots were fixed in equal volumes of $4 \%$

106 glutaraldehyde in $0.1 \mathrm{M}$ phosphate buffered saline ( $\mathrm{pH}$ 7.4), placed on poly-L-lysine coated 12

107 mm coverslips, and incubated for 5 minutes. The coverslips were washed with water and

108 dehydrated through increasing concentrations of ethanol $(25 \%, 50 \%, 75 \%, 95 \%)$ for five minutes

109 in each followed by three 5-minute changes in 100\% ethanol. Samples were dried in a Leica

110 Microsystems (model EM CPD300) critical point drier using liquid carbon dioxide as the

111 transitional field. Lastly, samples were mounted on aluminum stubs using epoxy glue (System

112 Three Quick Cure 5, System Three Resins, Inc, Lacey, Washington, USA) and coated with

113 osmium ( 10 mm thickness) using a NEOC-AT osmium coater (Meiwafosis Co., Ltd, Tokyo,

114 Japan). Imaging was performed using a JEOL 7500F scanning electron microscope. 
To evaluate MV morphology and purity, transmission electron microscopy (TEM) was

116 performed on purified vesicles as described (19). MVs were fixed in 4\% paraformaldehyde,

117 loaded onto formvar-carbon coated grids, and counterstained with $2.5 \%$ glutaraldehyde and $0.1 \%$

118 uranyl acetate in PBS. Samples were imaged using a JEOL 1400 Flash transmission electron

119 microscope.

\section{Quantification of vesicle production}

Nanoparticle tracking analysis was performed to quantify MVs produced by each strain

$123(\mathrm{n}=8-9$ replicates per strain) using a NanoSight NS300 (Malvern Panalytical Westborough, MA,

124 USA) equipped with an automated syringe sampler as described previously $(19,21)$. For each

125 sample, MVs were diluted in phosphate buffered saline (1:100 - 1:1000) and injected with a flow

126 rate of 50. Once loaded, five 20-second videos were recorded at a screen gain of 1 and camera

127 level of 13. After capture, videos were analyzed at a screen gain of 10 and a detection threshold

128 of 4 and data were subsequently exported to a CSV file for analysis using the R package

129 tidyNano (accessed via: https://nguyens7.github.io/tidyNano) (21). Total MV counts were

130 normalized by dividing by the colony forming units (CFUs) of each original bacterial culture.

\section{Proteomics}

133 Proteomic LC-MS/MS analysis of MVs was performed in duplicate or triplicate by the

134 Proteomics Core at the Michigan State University Research Technology Support Facility

135 (RTSF). Protein concentrations of purified MVs were determined using the Pierce Bicinchoninic

136 Acid Assay (ThermoFisher Scientific, Waltham, Massachusetts) supplemented with 2\% SDS in

137 water to reduce the background signal from excess lipids contained within the vesicles. MVs (1.5 
$138 \mu \mathrm{g})$ were concentrated into a single band in a 4-20\% Tris-Glycine SDS-PAGE gel (BioRad,

139 Hercules, CA) that was fixed and stained using colloidal Coomassie blue (22).

140 Protein bands were excised from the gels and stored in $5 \%$ acetic acid at $4{ }^{\circ} \mathrm{C}$. Prior to

141 analysis, in-gel trypsin digest and peptide extraction were performed. Briefly, gel bands were

142 dehydrated twice using 100\% acetonitrile and incubated with $10 \mathrm{mM}$ dithiothreitol in $100 \mathrm{mM}$

143 ammonium bicarbonate $\left(\mathrm{pH} \sim 8.0\right.$ ) at $56^{\circ} \mathrm{C}$ for 45 minutes. Bands were incubated in the dark with

$14450 \mathrm{mM}$ iodoacetamide in $100 \mathrm{mM}$ ammonium bicarbonate for 20 minutes followed by another

145 dehydration. Sequencing grade modified trypsin $(0.01 \mu \mathrm{g} / \mathrm{uL}$ in $50 \mathrm{mM}$ ammonium bicarbonate $)$

146 was added to each gel band and incubated at $37^{\circ} \mathrm{C}$ overnight. Peptides extracted by bath

147 sonication (in 60\% acetonitrile, $1 \%$ trichloroacetic acid solution) were vacuum dried and re-

148 suspended (in $2 \%$ acetonitrile $/ 0.1 \%$ trifluoroacetic) prior to separation using a Thermo

149 ACCLAIM C18 trapping column. Peptides were sprayed onto a ThermoFisher Q-Exactive HFX

150 mass spectrometer for 90 minutes; the top 30 ions per survey were analyzed further using high

151 energy induced dissociation. MS/MS spectra were converted into peak lists using Mascot

152 Distiller v2.7.0 and searched against a SwissProt database containing all GBS sequences

153 available through the National Center for Biotechnology Information (NCBI; accessed

154 2/08/2019). Contaminants were identified using Mascot searching algorithm v2.7.0, while

155 protein identities were validated using Scaffold v4.11.1.

\section{Data analysis}

To compare MV proteins between strains, proteomic data from all strains were compiled 159 and normalized for inter-experimental variability using Scaffold. Only proteins with a minimum 160 of two identified peptides falling above a $1 \%$ false discovery rate and $95 \%$ protein threshold, 
161 were considered for downstream analysis. Proteins identified as contaminants (via the Mascot

162 searching algorithm $\mathrm{v}$ 2.6.0) were removed, whereas proteins identified in both replicates for at

163 least one strain were classified as MV-associated. Subcellular localization analysis was

164 performed using pSORTdb (https://db.psort.org) with protein localization data for GBS strain

165 2603VR (downloaded from pSORTdb on 3/6/2021). Data visualization and statistical analyses

166 were performed using R version 4.1.0 (https://www.R-project.org). Principle component analysis

167 (PCA) was performed and visualized using the prcomp and fviz_pca functions, respectively.

168 Hierarchical clustering was performed using the pheatmap function and clustered using

169 Euclidean distances. Shapiro tests were used to determine whether data followed a normal

170 distribution and Student t-test (two-sided) or Kruskal-Wallis one-way analysis of variance

171 (ANOVA), in combination with the Dunn's posthoc test, were utilized to test for differences

172 between groups. Multiple hypothesis testing was corrected using Benjamini-Hochberg or

173 Bonferroni correction when necessary.

176 RESULTS

178 MV production varies across GBS strains

179 Visualization using SEM revealed abundant production of MVs by all six strains; these

180 MVs were closely associated with bacterial cells (Figure 1A-B, Figure S1). Within a given

181 culture, however, some cells displayed a relatively greater number of MVs on the cell surface

182 (Figure S1). While rare, these "hyper-producers" were observed in different samples and strains.

183 In addition, TEM revealed that MVs displayed a spherical morphology containing a lipid bilayer 
184 and slightly electron dense interior (Figure 1C-D, Figure S2), which is typical of bacterial-

185 derived MVs $(14,15)$.

Because electron microscopy suggested differences in MV production across strains, we

187 used NanoSight analysis to quantify MV size and production. MVs from each of the six strains

188 displayed a uniform size distribution, ranging between 100 and $200 \mathrm{~nm}$ (Figure 2A). Similar size

189 distributions were also observed by ST. For MV quantification, total MV counts were

190 normalized to the number of CFUs in the original bacterial cultures. Among the six strains, the

191 average number of MVs/CFU was 0.108 with a range of 0.048-0.206 MVs/CFU; however,

192 considerable variation was detected between strains (Figure 2B). Although no difference in MV

193 quantity was observed in colonizing versus invasive strains belonging to ST-1 or ST-17, the ST-

1941 strains produced significantly fewer MVs relative to the ST-17 strains (Figure S3; $\mathrm{p}<0.0001$ ).

195 While the colonizing ST-12 (cpsII) GB653 strain produced similar vesicle quantities as the two

196 ST-17 (cpsIII) strains, the invasive ST-12 (cpsII) isolate, GB1455, produced significantly more

197 MVs than all other strains examined $(\mathrm{p}<0.05)$. By contrast, the colonizing ST-1 (cpsV) isolate,

198 GB20, produced significantly fewer MVs compared to the strains representing all other STs (p

$199<0.05)$.

200

201 The MV proteome differs across GBS strains.

202

Proteomics of purified MVs identified 643 total proteins among the six isolates with an

203 average of 458 proteins per strain and range of 239-614 proteins per strain (Table S1A). Of note,

204 the number of unique proteins varied by strain. MVs from ST-1 strains, for instance, had fewer

205 unique proteins relative to the other STs with an average of 281 proteins compared to 601 and

206493 for the ST-12 and ST-17 strains, respectively. Regardless of ST, however, pSORTdb 
207 predicted numerous proteins to be membrane (12-17\%) and cell wall (2-11\%) localized, while

208 22-52\% were predicted to be localized in the cytoplasm (Figure 3A). Although many proteins

209 had a predicted subcellular localization, a large proportion of proteins had unidentified or

210 unpredicted subcellular localization.

Among the total proteins detected, 62 were found in all biological replicates for the six

212 strains (Table S1B). These proteins did not vary in spectral abundance between STs and

213 therefore represent the shared MV proteome. Of these 62 proteins, 11 were highly abundant with

214 a mean spectral count greater than 50 (Table S1C). Putative, uncharacterized transporters

215 constituted many of these shared proteins, accounting for 39-44\% of membrane protein spectral

216 counts. In addition, 19-25\% of spectral counts were predicted to have a membrane associated

217 subcellular localization (Figure 3B).

218 Next, we examined whether these proteins were strain-specific or if they were shared in

219 the six strains examined. Of all 643 proteins detected, $192(29.9 \%)$ were detected in at least one

220 biological replicate for all six strains regardless of the clinical phenotype or ST (Figure 4). In

221 addition, $124(19.28 \%)$ proteins were shared by the four ST-12 and ST-17 strains but were

222 absent in the ST-1 strains, suggesting that the ST-1 MVs have a unique protein composition.

223 While a minor proportion of proteins were ST- or strain specific, none were shared by all

224 invasive or all colonizing strains.

225 We next considered the relationship between protein composition and strain

226 characteristics using PCA. Even though the protein composition of MVs from invasive and

227 colonizing strains overlapped, it was segregated by ST (Figure 5), though some overlap was

228 observed between the ST-12 confidence ellipse and those for other STs. No overlap, however,

229 was seen between the ST-1 and ST-17 strains, highlighting their distinct proteomes. This distinct 
230 clustering was not observed when the relationship between protein composition and clinical

231 phenotype was analyzed (Figure S4), with invasive and colonizing samples displaying a high

232 degree of overlap with little to no separation of their respective confidence ellipses.

233 Hierarchical clustering of the protein data further demonstrated that MVs from strains

234 belonging to the same ST had similar protein profiles forming distinct clusters by ST regardless

235 of the clinical phenotype (Figure 6). Specifically, proteins from the ST-12 and ST-17 strains

236 formed a distinct branch in the phylogeny that was separate from the ST-1 proteins, indicating

237 that their protein composition was more similar to each other than to ST-1 strains. This

238 observation supports the PCA, showing a higher degree of overlap between ST-12 and ST-17

239 strains compared to ST-1 strains. Nonetheless, ST-12 and ST-17 strains were still

240 distinguishable, with distinct nodes forming based on protein composition, indicating their

241 divergent composition. This analysis further revealed that ST-1 strains lacked several proteins

242 that were highly abundant in both the ST-12 and ST-17 strains. To a lesser degree than ST-1

243 MVs, we observed that several highly abundant proteins found among the ST-17 strains were

244 entirely absent in ST-12 strains.

246 Key virulence factors were differentially abundant in MVs across GBS lineages.

247 To determine which proteins contributed most to the segregation observed in the PCA

248 and hierarchical clustering analyses, we more thoroughly examined the 335 proteins that were

249 significantly enriched in at least one ST (Table S2). Notably, several purported virulence factors

250 including the C5a peptidase, hyaluronidase, and sialidase were highly enriched in a ST-

251 dependent manner (Figure 7). Both the hyaluronidase and C5a peptidase were significantly more

252 abundant in the two ST-17 strains compared to the ST-1 and ST-12 strains, whereas the sialidase 
253 was detected at significantly higher levels in ST-1 versus ST-12 strains. Several proteins of

254 unknown function were also among the most highly abundant and differentially enriched

255 proteins detected. One hypothetical protein, for instance, was significantly more abundant in the

256 ST-1 strains relative to strains representing the other two lineages (Figure 7). Similarly, another

257 hypothetical protein was more abundant in the ST-12 strains (Figure S5); however, considerable

258 variation was observed across replicates. Numerous phage-associated proteins including a holin

259 and capsid protein, were also detected and found to be more abundant in the ST-17 strains along

260 with several proteins associated with cell division (Figure S6). For example, the average

261 abundance of cell division proteins FtsE, FtsQ, FtsZ, and FtsY, was significantly greater in the

262 two ST-17 strains compared to those from other lineages. Differences in proteins linked to cell

263 wall modification such as penicillin-binding proteins and capsule biosynthesis proteins, were

264 also detected (Figure S7).

\section{DISCUSSION}

268 Current knowledge regarding GBS derived MVs is restricted to one clinical strain (14,

269 15) and hence, we sought to examine MV production and composition in a set of clinical strains

270 with different traits. While no clear association was observed between clinical phenotype and the

271 production or composition of MVs, we have demonstrated that the GBS MV proteome is ST-

272 dependent. The same was observed for MV production, though some variation was noted

273 between strains of the same ST. Together, these data indicate that GBS MVs have strain-

274 dependent functions that could impact survival in hosts, immunomodulation, and virulence. 
This study expands our current knowledge of GBS MVs by highlighting their potential

276

277 immunomodulatory virulence factors including C5a peptidase, hyaluronidase, and sialidase (23-

278 25). The bifunctional C5a peptidase has been shown to promote the degradation of the

279 proinflammatory complement component (C5a) while simultaneously promoting bacterial

280 invasion into host cells $(23,24)$. MVs from both ST-17 (cpsIII) strains examined herein

281 contained high levels of C5a peptidase, whereas ST-1 and ST-12 strains lacked this protein.

282 Intriguingly, ST-17 strains were previously shown to possess distinct virulence gene profiles as

283 well as unique alleles of $s c p B$ encoding the C5a peptidase $(26,27)$, suggesting that ST-17 strains

284 may be primed to cause invasive infections. This suggestion is in line with epidemiological data

285 showing that ST-17 strains are important for invasive disease in adults and neonates $(6,8,9)$ as

286 well as mechanistic studies showing an enhanced ability to attach to gestational tissues, induce

287 stronger proinflammatory responses, and persist inside macrophages (10-12). Nonetheless, it is

288 important to note that our clinical definitions of "invasive" versus "colonizing" strain types may

289 not be representative of each strain population. Although strains isolated from an active infection

290 clearly demonstrate "invasive" potential, it is possible that strains designated as "colonizing"

291 could also cause an infection in specific circumstances and host environments.

Although sialidases have no known role in GBS pathogenesis (25), these proteins were

293 shown to be immunomodulatory in other bacterial species $(28,29)$ while simultaneously

294 promoting biofilm production and metabolism of host sugars $(30,31)$. The presence and

295 abundance of sialidase was variable: the ST-1 and ST-17 MVs all contained sialidase, but the

296 ST-12 MVs lacked it. In two prior studies examining GBS MVs produced by a ST-7 strain,

297 A909, neither C5a peptidase nor sialidase were identified (14, 15), further highlighting 
differences across strains. However, we cannot rule out the possibility that the abundance of

299 these virulence factors was beneath the detection limit in those studies. Similarly, the previous analysis of GBS MVs highlighted the importance of hyaluronidase (14). This

301 immunomodulatory factor has previously been shown to promote ascending infection, degrade

302 host extracellular matrix components, and dampen the host immune response (24). While we

303 also found high levels of hyaluronidase in the ST-17 MVs examined, our results further show

304 that the ST-12 and ST-1 MVs contained significantly lower amounts of this protein.

It is also important to note that multiple uncharacterized and hypothetical proteins were

306 detected. Previous reports have demonstrated that in gram positive species, roughly $30-60 \%$ of

307 all MV proteins map to the cytoplasm $(32,33)$. While our results are consistent with this

308 observation showing $\sim 22-52 \%$ of all proteins mapping to the cytoplasm, roughly $25-41 \%$ of the

309 GBS MV proteins had an unidentifiable subcellular localization. Similar trends of ST-dependent

310 enrichment of several hypothetical proteins were observed, with these representing some of the

311 most highly abundant proteins. Although some uncharacterized proteins, such as those classified

312 as putative $\mathrm{ABC}$ transporters, have predicted functions, their role in vesicle function or virulence

313 is currently unknown. Future analyses must be undertaken to identify which proteins play a role

314 in MV associated pathogenesis.

315 Through this study, we have also identified a shared proteome among MVs from

316 phylogenetically distinct GBS strains. In total, 62 proteins were consistently found within GBS

317 MVs regardless of the ST. Indeed, over 17\% of these shared proteins were highly abundant,

318 indicating that they may be important for MV functionality. Even though many of these proteins

319 have yet to be characterized, we identified an abundance of transporter proteins in MVs 
320 suggesting a potential role in MV function. Separate of functionality, these shared proteins may

321 be of value as potential MV markers in future studies.

322 While various mechanisms have been proposed for the biogenesis of gram positive MVs,

323 those mechanisms important for GBS MV biogenesis are unclear $(13,34)$. Our data demonstrate

324 that diverse GBS strains produce MVs with consistent size distributions, indicating that GBS

325 MV production is ubiquitous. Purported mechanisms of MV biogenesis include phage mediated

326 biogenesis $(35,36)$, membrane budding during division $(37)$, and cell wall remodeling $(13,38)$.

327 In line with these mechanisms, our proteomics analysis revealed the presence of phage

328 associated proteins, division septum-associated proteins and cell wall-modifying enzymes.

329 Several of these proteins were also differentially abundant, with some proteins being more highly

330 enriched in certain STs. For instance, phage proteins were enriched in ST-17 strains but were

331 nearly absent in ST-12 and ST-1 strains. Although we observed similar enrichment of cell

332 division proteins in ST-12 and ST-17 strains relative to ST-1, cell wall modifying proteins were

333 most abundant in the ST-17 strains. Taken together, these data indicate that MVs are produced

334 by diverse strains with varying traits; however, the mechanisms for MV biogenesis appear to be

335 strain dependent. Additional studies are needed to test this hypothesis.

Although our study has enhanced our understanding of the proteomic composition of

337 GBS MVs, it has a few limitations. Because strains of each GBS lineage possess the same

338 capsule (cps) type, it is difficult to differentiate between ST versus cps effects. Another concern

339 when dealing with MVs is the presence of non-vesicular contaminants. In some eukaryotic and

340 prokaryotic systems where the composition of MVs is well defined, markers are used to assess

341 purity (39-41). Due to the relatively unknown composition of GBS MVs, however, we were

342 unable to target specific markers to evaluate the purity. Rather, we relied on size exclusion 
343 chromatography followed by TEM to further remove non-vesicular proteins from each MV

344 preparation. While we likely have some contaminant proteins, the purity of our preparations

345 exceeds those performed in prior GBS studies $(14,15)$ and mimics protocols optimized for

346 removing extravesicular macromolecules from Gram positive MVs (14, 15, 42, 43). Indeed,

347 studies in Staphylococcus aureus and Streptococcus mutans have confirmed the presence of

348 similar proportions of cytoplasmic and extracellular proteins within MVs $(32,33)$. Further, while

349 our study has greatly enhanced our understanding of GBS MV composition, it is known that

350 other macromolecules are present within MVs (14). Whether these macromolecules display ST

351 dependent composition is unclear; however, given these data further studies are warranted.

352 In summary, this comprehensive analysis of GBS MVs from strains representing three

353 phylogenetically distinct lineages demonstrates strain dependent composition and production of

354 MVs. Our data further demonstrate that MVs carry both known virulence factors and other

355 proteins of unknown function in variable abundance between strains, suggesting that they may

356 have altered functionality or ability to promote virulence. Follow up studies elucidating virulence

357 and immunomodulatory properties of diverse strains of GBS MVs are therefore warranted,

358 particularly given the high level of variation in protein composition observed among only these

359 six strains. Taken together, these findings further highlight the importance of strain variation in

360 GBS pathogenesis and shed light on the potential role of MVs in virulence. 


\section{Acknowledgments}

367 We would like to thank Dr. H. Dele Davies for sharing the bacterial strains and Drs. Sean L.

368 Nguyen and Soo H. Ahn for helpful conversations and assistance with data analysis. We also

369 thank Karla Vasco for assisting with the hierarchical clustering heatmap analysis as well as

370 Alicia Withrow, Carol Flegler, and Douglas Whitten for their assistance with TEM, SEM, and

371 proteomics analysis, respectively.

372

\section{$373 \quad$ Funding}

374 This work was funded by the National Institutes of Health (NIH; AI154192 to S.D.M and

375 M.G.P.) with additional support provided by AI134036 to D.M.A and HD090061 to J.A.G. and

376 BX005352 from the Office of Research, Department of Veterans Affairs. Graduate student

377 support for C.R.M. was provided by the Reproductive and Developmental Science Training

378 Program funded by the NIH (T32 HDO87166) as well as the Eleanor L. Gilmore Endowed

379 Excellence Award.

\section{Data Availability}

382 Raw proteomic data was submitted to the MassIVE database (massive.ucsd.edu). Data can be 383 accessed via https://doi.org/doi:10.25345/C5RC1H or ftp://massive.ucsd.edu/MSV000087985/. 


\section{References}

390 1. Verani JR, McGee L, Schrag SJ. 2010. Prevention of perinatal group B streptococcal

391 disease--revised guidelines from CDC, 2010. Morb Mortal Wkly Rep 59:1-36.

392 2. Doran KS, Nizet V. 2004. Molecular pathogenesis of neonatal group B streptococcal 393 infection: no longer in its infancy. Mol Microbiol 54:23-31.

394 3. Edwards MS, Baker CJ. 2005. Group B streptococcal infections in elderly adults. Clin $395 \quad$ Infect Dis 41:839-47.

396 4. Aronoff DM, Blaser MJ. 2020. Disturbing the neonatal microbiome is a small price to 397 pay for preventing early-onset neonatal group B Streptococcus disease: AGAINST: 398 Against relying on antibiotics to prevent early-onset neonatal group B Streptococcus 399 disease. BJOG 127:229.

400 5. Bianchi-Jassir F, Paul P, To KN, Carreras-Abad C, Seale AC, Jauneikaite E, Madhi SA,

401 Russell NJ, Hall J, Madrid L, Bassat Q, Kwatra G, Le Doare K, Lawn JE. 2020.

402 Systematic review of group B streptococcal capsular types, sequence types and surface $403 \quad$ proteins as potential vaccine candidates. Vaccine 38:6682-6694.

404 6. Jones N, Bohnsack JF, Takahashi S, Oliver KA, Chan MS, Kunst F, Glaser P, Rusniok C, 405 Crook DW, Harding RM, Bisharat N, Spratt BG. 2003. Multilocus sequence typing 406 system for group B Streptococcus. J Clin Microbiol 41:2530-6.

407 7. Manning SD, Lewis MA, Springman AC, Lehotzky E, Whittam TS, Davies HD. 2008. 408 Genotypic diversity and serotype distribution of group B Streptococcus isolated from 409 women before and after delivery. Clin Infect Dis 46:1829-37.

410 8. Flores AR, Galloway-Peña J, Sahasrabhojane P, Saldaña M, Yao H, Su X, Ajami NJ, 411 Holder ME, Petrosino JF, Thompson E, Margarit Y Ros I, Rosini R, Grandi G, 
415 9. Manning SD, Springman AC, Lehotzky E, Lewis MA, Whittam TS, Davies HD. 2009. meningitis in Canada. J Clin Microbiol 47:1143-8.

418 10. Korir ML, Knupp D, LeMerise K, Boldenow E, Loch-Caruso R, Aronoff DM, Manning SD. 2014. Association and virulence gene expression vary among serotype III group B Streptococcus isolates following exposure to decidual and lung epithelial cells. Infect Immun 82:4587-95.

422 11. Flaherty RA, Borges EC, Sutton JA, Aronoff DM, Gaddy JA, Petroff MG, Manning SD. cytokine responses. PLoS One 14:e0222910.

425 12. Korir ML, Laut C, Rogers LM, Plemmons JA, Aronoff DM, Manning SD. 2017.

426 Differing mechanisms of surviving phagosomal stress among group B Streptococcus

427 strains of varying genotypes. Virulence 8:924-937.

428 13. Brown L, Wolf JM, Prados-Rosales R, Casadevall A. 2015. Through the wall:

429 extracellular vesicles in Gram-positive bacteria, mycobacteria and fungi. Nat Rev

$430 \quad$ Microbiol 13:620-30.

431 14. Surve MV, Anil A, Kamath KG, Bhutda S, Sthanam LK, Pradhan A, Srivastava R, Basu 
435 15. Armistead B, Quach P, Snyder JM, Santana-Ufret V, Furuta A, Brokaw A, Rajagopal L. 2021. Hemolytic membrane vesicles of group B Streptococcus promote infection. J Infect Dis 223:1488-1496.

438 16. Davies HD, Adair C, McGeer A, Ma D, Robertson S, Mucenski M, Kowalsky L, Tyrell G, Baker CJ. 2001. Antibodies to capsular polysaccharides of group B Streptococcus in pregnant Canadian women: relationship to colonization status and infection in the

442 17. Spaetgens R, DeBella K, Ma D, Robertson S, Mucenski M, Davies HD. 2002. Perinatal antibiotic usage and changes in colonization and resistance rates of group B Streptococcus and other pathogens. Obstet Gynecol 100:525-33.

445 18. Klimentová J, Stulík J. 2015. Methods of isolation and purification of outer membrane vesicles from gram-negative bacteria. Microbiol Res 170:1-9.

447 19. Nguyen SL, Ahn SH, Greenberg JW, Collaer BW, Agnew DW, Arora R, Petroff MG. 2021. Integrins mediate placental extracellular vesicle trafficking to lung and liver in vivo. Sci Rep 11:4217.

450 20. Chutkan H, Macdonald I, Manning A, Kuehn MJ. 2013. Quantitative and qualitative preparations of bacterial outer membrane vesicles. Methods Mol Biol 966:259-72.

452 21. Nguyen SL, Greenberg JW, Wang H, Collaer BW, Wang J, Petroff MG. 2019.

453 Quantifying murine placental extracellular vesicles across gestation and in preterm birth 454 data with tidyNano: A computational framework for analyzing and visualizing 455 nanoparticle data in R. PLoS One 14:e0218270.

456 22. Dyballa N, Metzger S. 2009. Fast and sensitive colloidal coomassie G-250 staining for 457 proteins in polyacrylamide gels. J Vis Exp. 30:1431. 
458 23. Cheng Q, Stafslien D, Purushothaman SS, Cleary P. 2002. The group B streptococcal

459 C5a peptidase is both a specific protease and an invasin. Infect Immun 70:2408-13.

460 24. Kolar SL, Kyme P, Tseng CW, Soliman A, Kaplan A, Liang J, Nizet V, Jiang D, Murali

461 R, Arditi M, Underhill DM, Liu GY. 2015. Group B Streptococcus evades host immunity

$462 \quad$ by degrading hyaluronan. Cell Host Microbe 18:694-704.

463 25. Yamaguchi M, Hirose Y, Nakata M, Uchiyama S, Yamaguchi Y, Goto K, Sumitomo T, 464 Lewis AL, Kawabata S, Nizet V. 2016. Evolutionary inactivation of a sialidase in group 465 B Streptococcus. Sci Rep 6:28852.

466 26. Brochet M, Couvé E, Zouine M, Vallaeys T, Rusniok C, Lamy MC, Buchrieser C, Trieu467 Cuot P, Kunst F, Poyart C, Glaser P. 2006. Genomic diversity and evolution within the $468 \quad$ species Streptococcus agalactiae. Microbes Infect 8:1227-43.

469 27. Springman AC, Lacher DW, Wu G, Milton N, Whittam TS, Davies HD, Manning SD. 470 2009. Selection, recombination, and virulence gene diversity among group B $471 \quad$ streptococcal genotypes. J Bacteriol 191:5419-27.

472 28. Sudhakara P, Sellamuthu I, Aruni AW. 2019. Bacterial sialoglycosidases in Virulence 473 and Pathogenesis. Pathogens 8:39.

474 29. Aruni W, Vanterpool E, Osbourne D, Roy F, Muthiah A, Dou Y, Fletcher HM. 2011.

475 Sialidase and sialoglycoproteases can modulate virulence in Porphyromonas gingivalis. $476 \quad$ Infect Immun 79:2779-91.

477 30. Hardy L, Jespers V, Van den Bulck M, Buyze J, Mwambarangwe L, Musengamana V, 478 Vaneechoutte M, Crucitti T. 2017. The presence of the putative Gardnerella vaginalis 479 sialidase A gene in vaginal specimens is associated with bacterial vaginosis biofilm. $480 \quad$ PLoS One 12:e0172522. 
481 31. Zaramela LS, Martino C, Alisson-Silva F, Rees SD, Diaz SL, Chuzel L, Ganatra MB, Gut bacteria responding to dietary change encode sialidases that exhibit preference for red meat-associated carbohydrates. Nat Microbiol 4:2082-2089.

33. Cao Y, Zhou Y, Chen D, Wu R, Guo L, Lin H. 2020. Proteomic and metabolic values. Appl Microbiol Biotechnol 104:9733-9748.

492 34. Briaud P, Carroll RK. 2020. Extracellular vesicle biogenesis and functions in grampositive bacteria. Infect Immun 88:e0433-20.

494 35. Toyofuku M, Cárcamo-Oyarce G, Yamamoto T, Eisenstein F, Hsiao CC, Kurosawa M, vesicle formation through peptidoglycan damage in Bacillus subtilis. Nat Commun 8:481.

497 36. Toyofuku M, Nomura N, Eberl L. 2019. Types and origins of bacterial membrane 498 vesicles. Nat Rev Microbiol 17:13-24.

499 37. Vdovikova S, Luhr M, Szalai P, Nygård Skalman L, Francis MK, Lundmark R, Engedal 500 N, Johansson J, Wai SN. 2017. A novel role of Listeria monocytogenes membrane 
502 38. Wang X, Thompson CD, Weidenmaier C, Lee JC. 2018. Release of Staphylococcus aureus extracellular vesicles and their application as a vaccine platform. Nat Commun 9:1379.

505 39. Sarker S, Scholz-Romero K, Perez A, Illanes SE, Mitchell MD, Rice GE, Salomon C. 2014. Placenta-derived exosomes continuously increase in maternal circulation over the 507 first trimester of pregnancy. J Transl Med 12:204.

508 40. Rompikuntal PK, Vdovikova S, Duperthuy M, Johnson TL, Åhlund M, Lundmark R, Oscarsson J, Sandkvist M, Uhlin BE, Wai SN. 2015. Outer membrane vesicle-mediated export of processed PrtV protease from Vibrio cholerae. PLoS One 10:e0134098.

511 41. Vanaja SK, Russo AJ, Behl B, Banerjee I, Yankova M, Deshmukh SD, Rathinam VAK. 2016. Bacterial outer membrane vesicles mediate cytosolic localization of LPS and caspase-11 activation. Cell 165:1106-1119.

514 42. Mehanny M, Koch M, Lehr CM, Fuhrmann G. 2020. Streptococcal extracellular membrane vesicles are rapidly internalized by immune cells and alter their cytokine release. Front Immunol 11:80.

517 43. Dauros Singorenko P, Chang V, Whitcombe A, Simonov D, Hong J, Phillips A, Swift S, 


\section{Figure 1: Electron microscopy of membrane vesicles (MVs) from overnight cultures post-}

523 purification. Overnight cultures of GBS strains were visualized by electron microscopy.

524 Representative images include the: A) invasive ST-1, cpsV (GB37) strain, and B) colonizing ST-

525 17, cpsIII (GB112) strain examined by scanning electron microscopy (SEM) at 10,000x

526 magnification with a minimum of 2 replicates per strain. SEM scale bars indicate $1 \mu \mathrm{m}$ length.

527 Representative transmission electron microscopy (TEM) images of MVs from the same C)

528 invasive and D) colonizing strains following purification using ultracentrifugation and size

529 exclusion chromatography (2-3 replicates per strain). TEM images were taken at a magnification

530 of $20,000 \mathrm{x}$ and the scale bars indicate a length of $200 \mathrm{~nm}$.

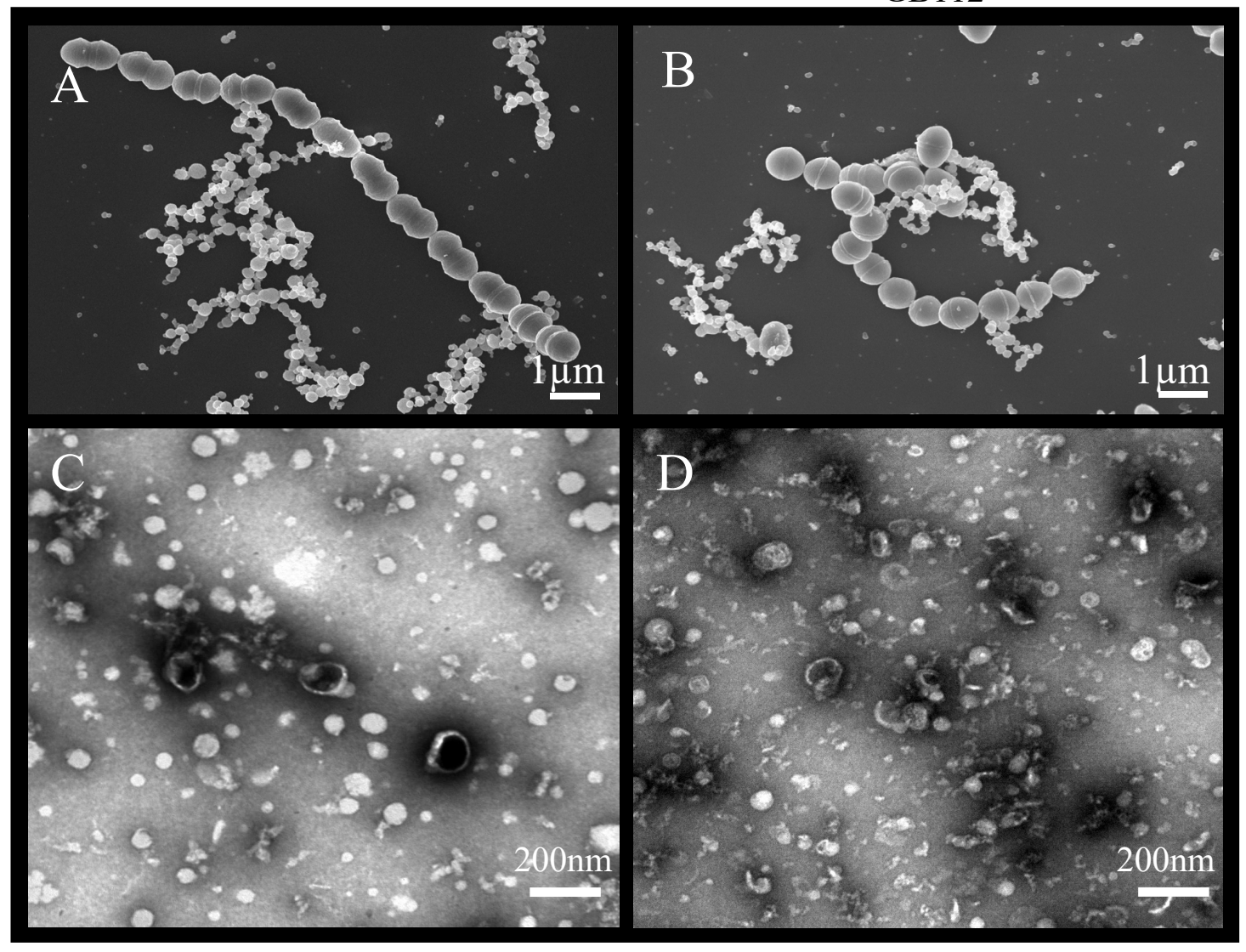


544 Figure 2: Quantitative assessment of membrane vesicle (MV) production across strains.

545 MVs were isolated by differential centrifugation and quantified using NanoSight analysis. The

546 vesicle A) size distribution and B) number per bacterial colony forming units (CFUs) are shown

547 for the invasive and colonizing strains by sequence type (ST). For panel B, the lines show the

548 mean across 8-9 biological replicates (indicated by colored dots). Shaded regions surrounding

549 the lines are the standard error of the mean and the black dots are outliers identified by

550 multiplying the interquartile range by 1.5 , which was used to extend the upper and lower

551 quartiles. Outliers were excluded from the analysis. Differences in production were assessed

552 using the Kruskal Wallis test followed by a posthoc Dunn's Test with a Benjamini-Hochberg

553 correction. *p-value $<0.05$ with higher production for all possible comparisons unless otherwise

554 indicated, while \# indicates a p-value $<0.05$ with lower production.
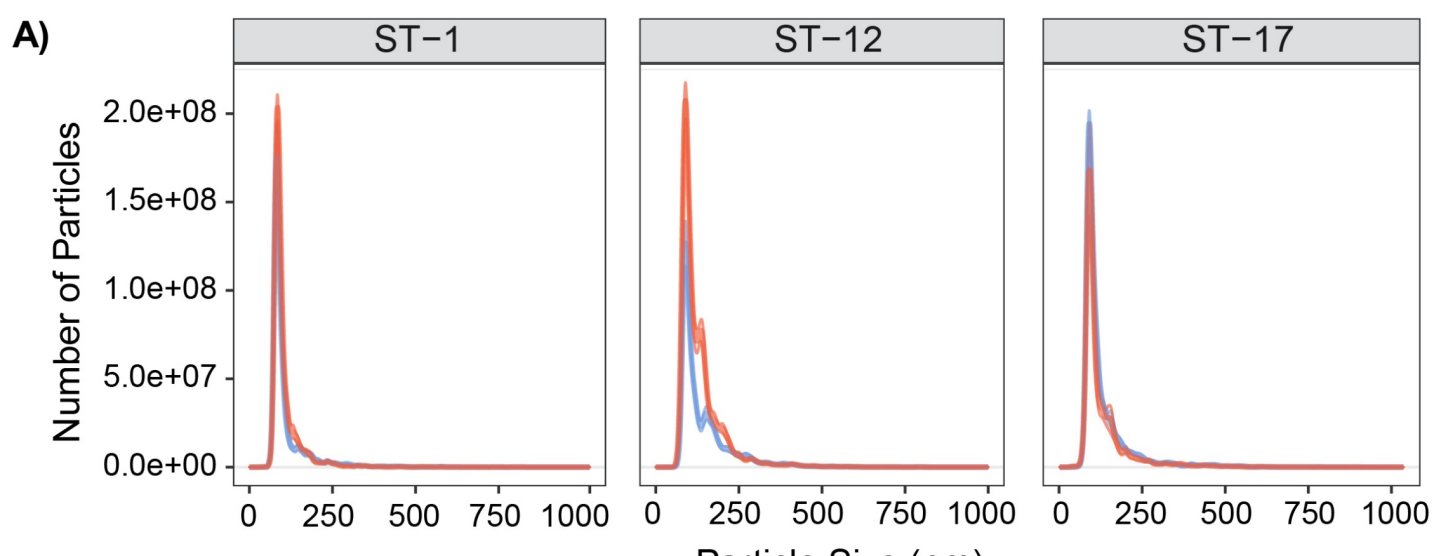

$$
\begin{aligned}
& \text { Phenotype } \\
& \text { - colonizing }
\end{aligned}
$$

- invasive

B)
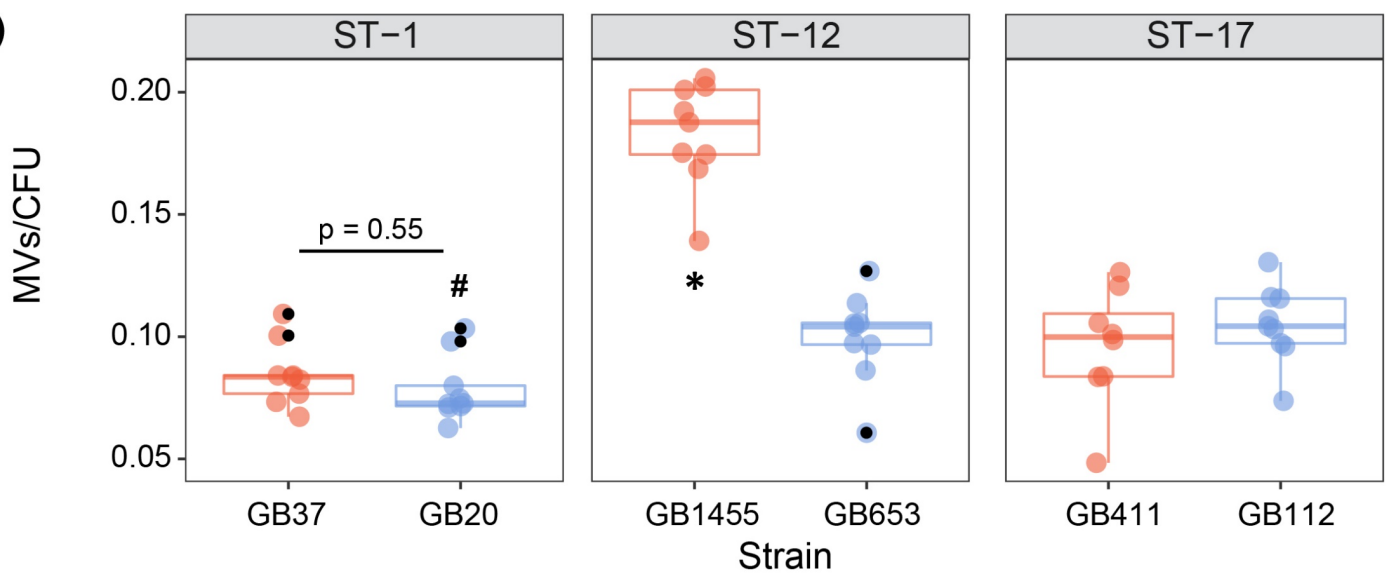

Phenotype

- colonizing

invasive 
555 Figure 3. Subcellular localization analysis of membrane vesicle (MV) proteomes. The

556 subcellular localization of A) all $643 \mathrm{MV}$ proteins identified, and B) a subset of 62 shared MV

557 proteins identified using a pSORTdb database for published Streptococcus agalactiae sequences

558 (accessed 3/3/21). Percentages were determined from mean spectral counts for a given sequence

559 type (ST).

A)

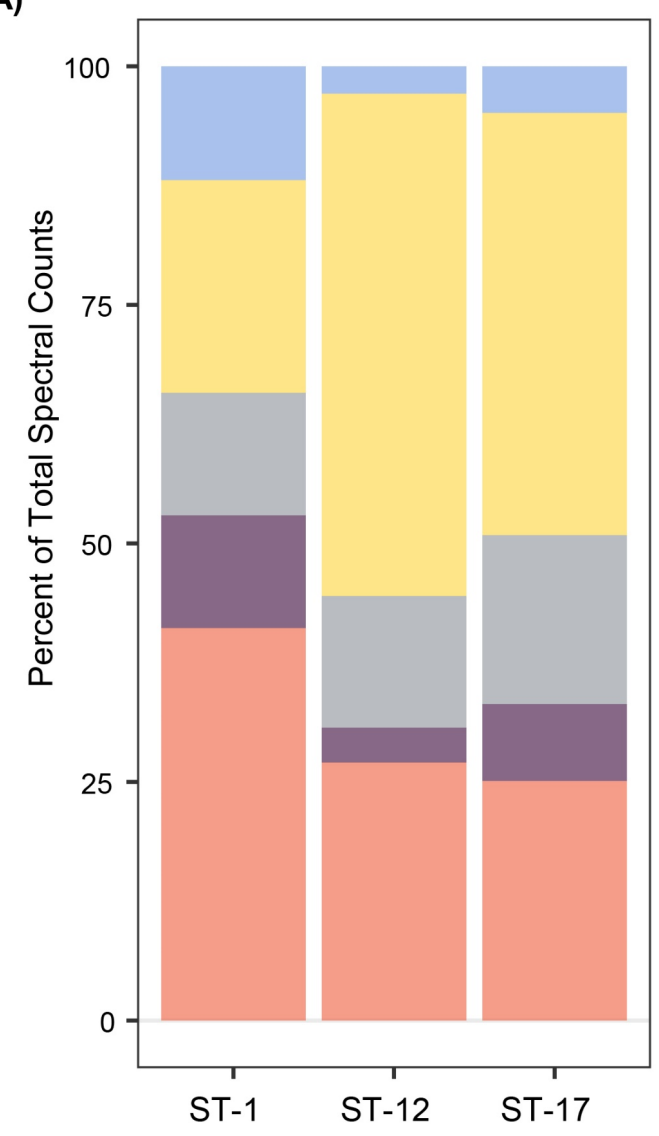

B)

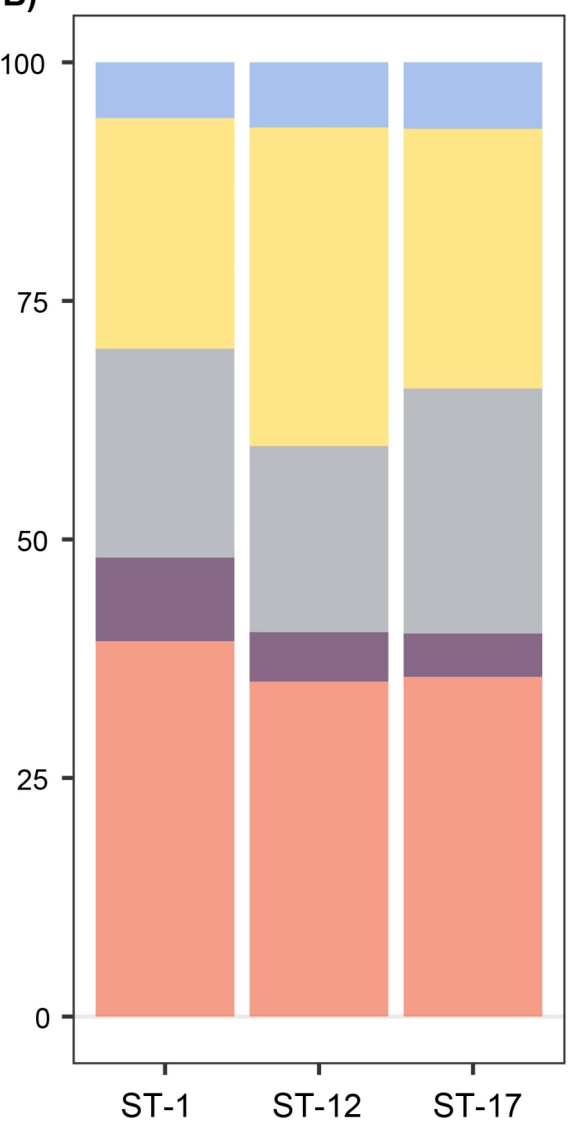

Subcellular localization

Cell wall

Cytoplasmic

Cytoplasmic membrane

Extracellular

Unknown 
Figure 4. Distribution of proteins detected in membrane vesicles (MVs) among six strains.

An Upset plot was generated to show the distribution of all 643 proteins detected across the six

567 GBS strains examined. The y-axis indicates the total number of proteins detected for a given set

568 of strains. Protein presence is defined as having a non-zero spectral count for a given protein in

569 at least one biological replicate for a specific strain. The matrix at the base of the plot shows the

570 strains ordered vertically by sequence type with filled bubbles indicating which strains are

571 positive for the number of proteins detected, and overlaid bars representing number of shared

572 proteins.

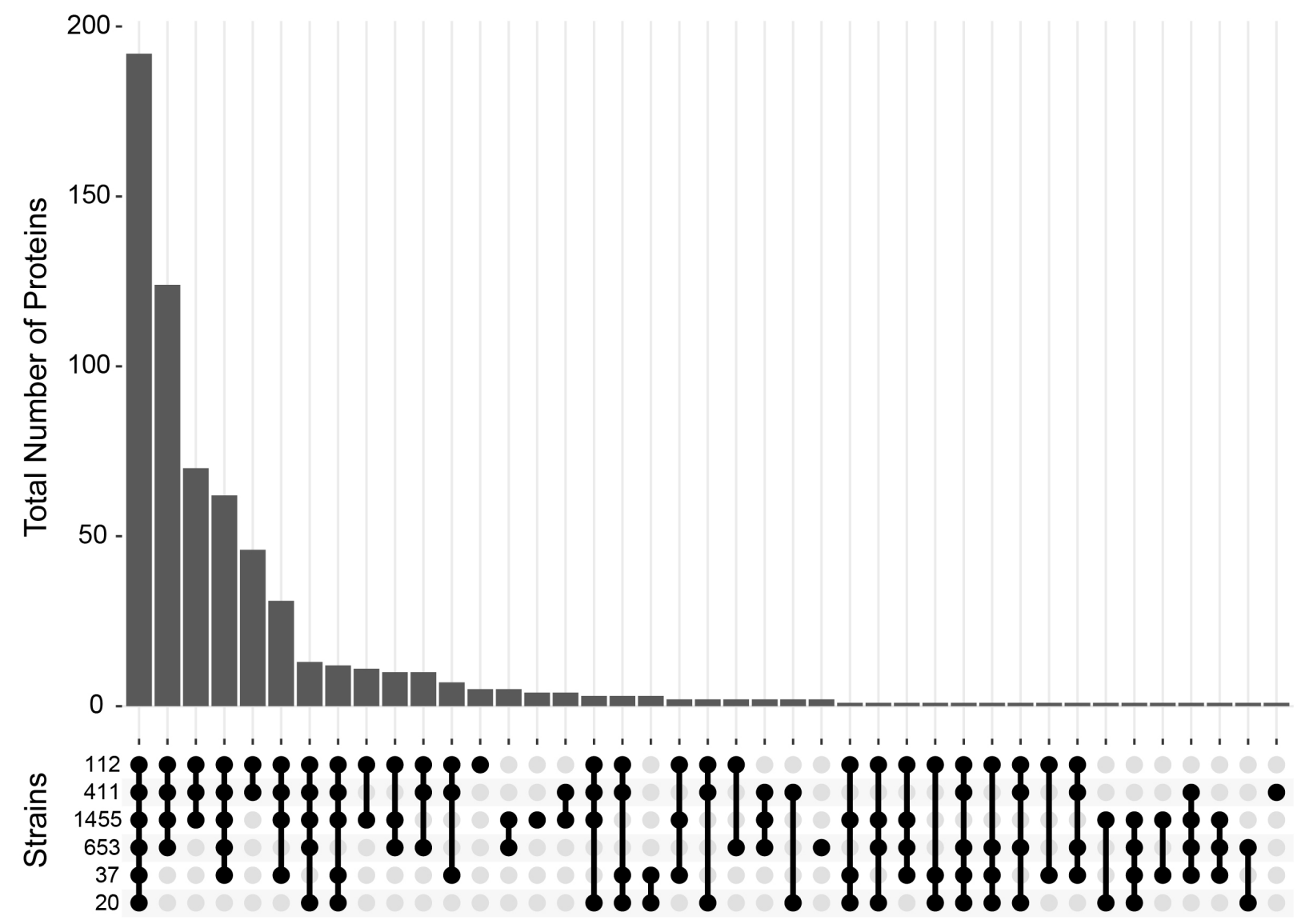




\section{Figure 5: Principal component analysis (PCA) reveals lineage-specific clustering of}

576 membrane vesicle (MV) proteomes. PCA of the MV proteomes produced by six strains

577 stratified by sequence type (ST). The large central dot of each ellipse represents the mean point

578 of the corresponding 95\% confidence ellipse, while the smaller points represent individual

579 proteomic samples. Confidence ellipses comprise $95 \%$ of the samples based on the underlying

580 distribution. Axes percentages represent the amount of variation accounted for by each principal

581 component (PC).

582

583

584

585

586

587

588

589

590

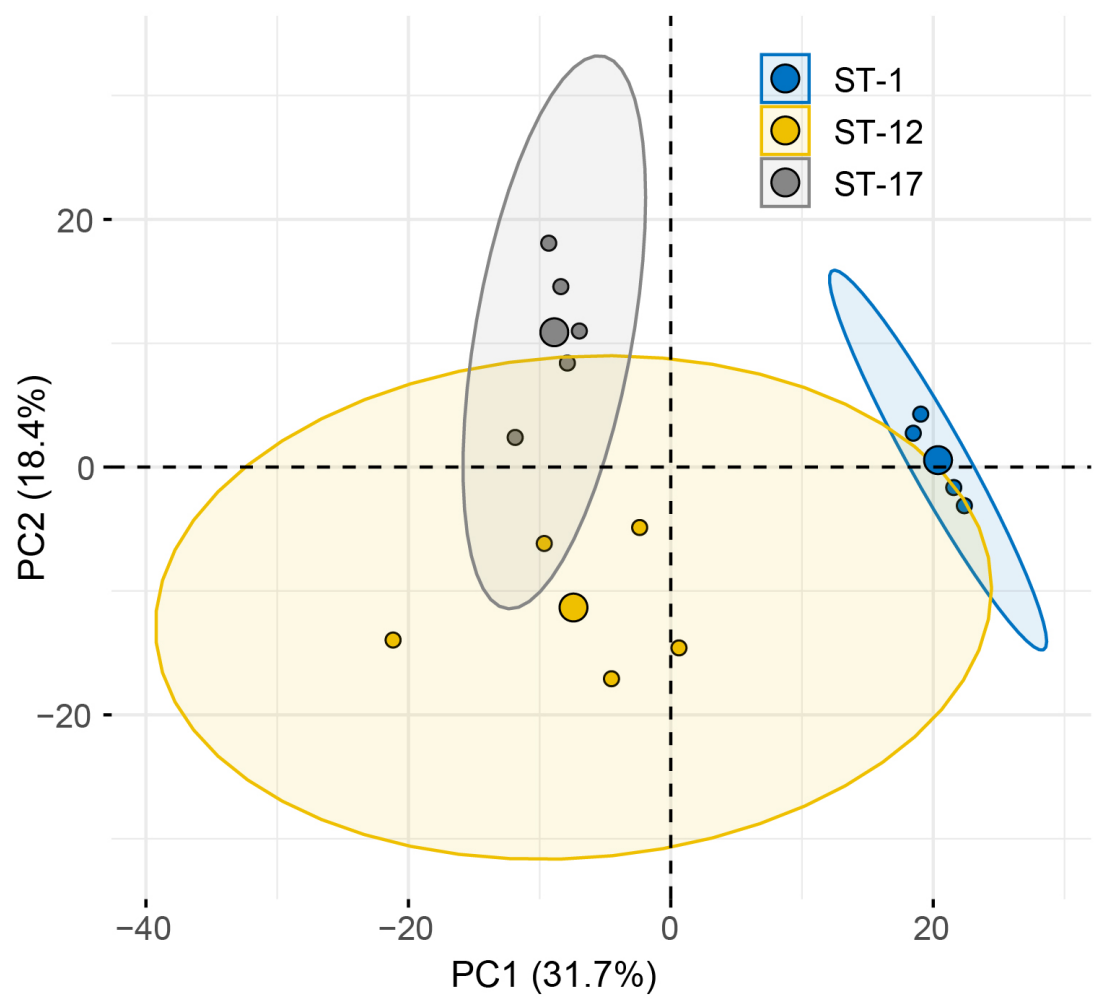

593

594

595

596 
602 individual boxes corresponding to the natural $\log (\mathrm{Ln})$ transformation of spectral counts for a

603 given protein of interest. Columns represent a single proteomic sample, which are color coded by

604 strain.

605

606

607

608

609

\section{In transformed spectral counts}

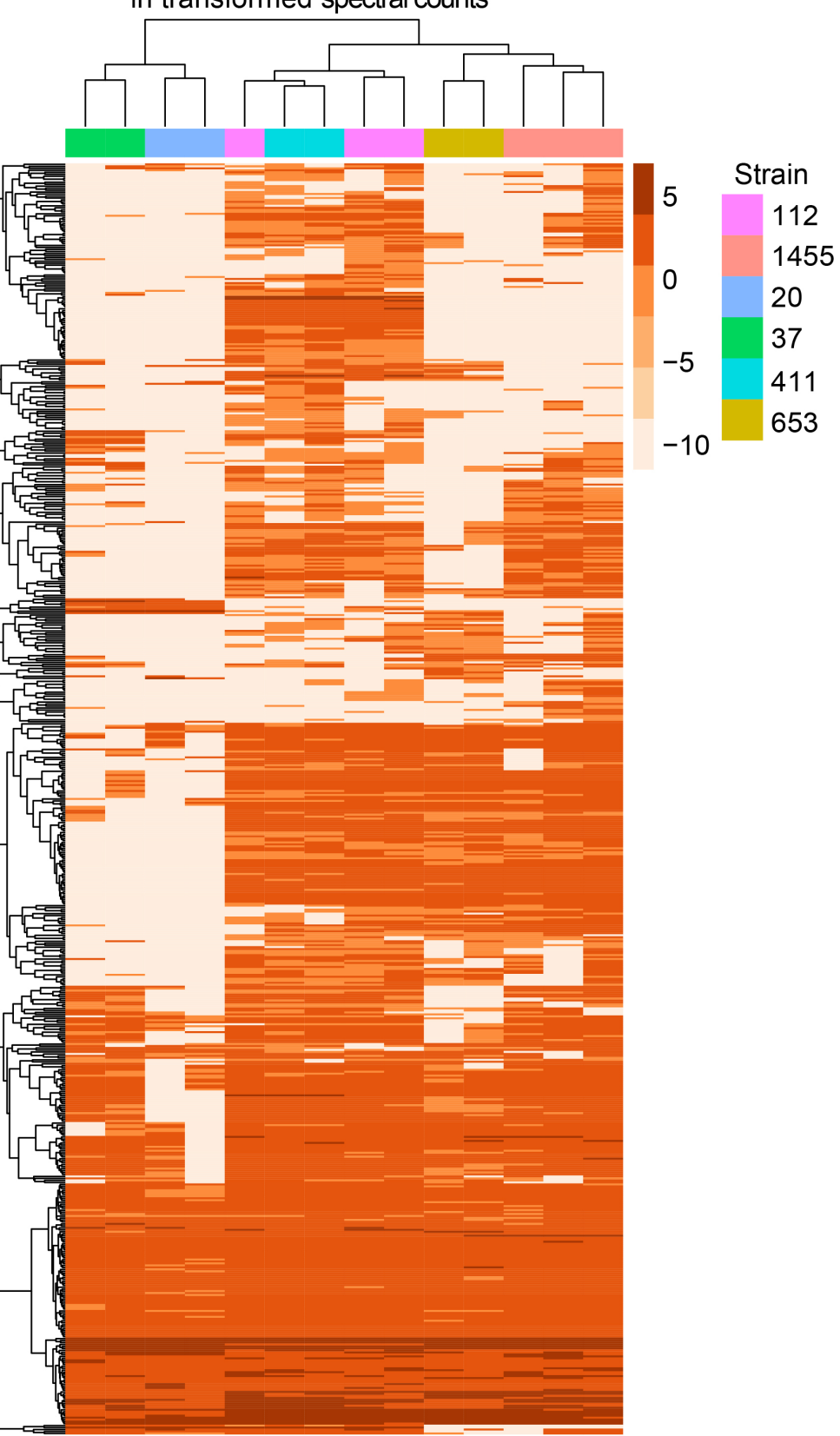




\section{Figure 7: Highly abundant proteins are present at variable levels in membrane vesicles}

622 (MVs). The spectral counts of specific proteins were plotted after stratifying by the sequence

623 type (ST). The median spectral count associated with each ST is represented within each box.

624 The black dots represent a single biological replicate for a given strain. Statistical comparison

625 was performed using a Kruskal Wallis test. Multiple pairwise comparisons were then made using

626 the pairw.kw function in R, which uses a conservative Bonferroni correction method to correct

627 for multiple hypothesis testing. Comparisons with p-values $<0.05$ are denoted with an asterisk.

628

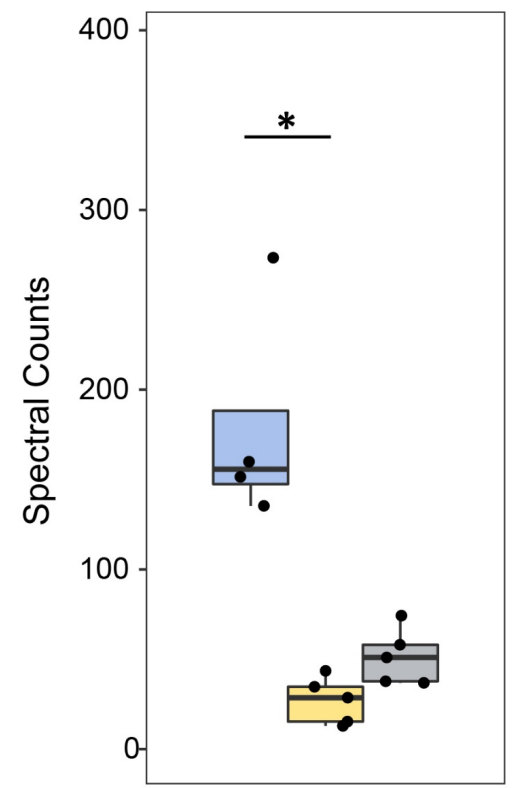

Hypothetical protein

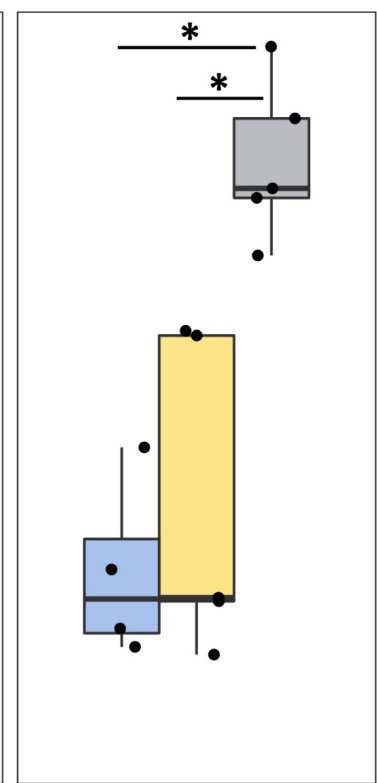

Hyaluronidase

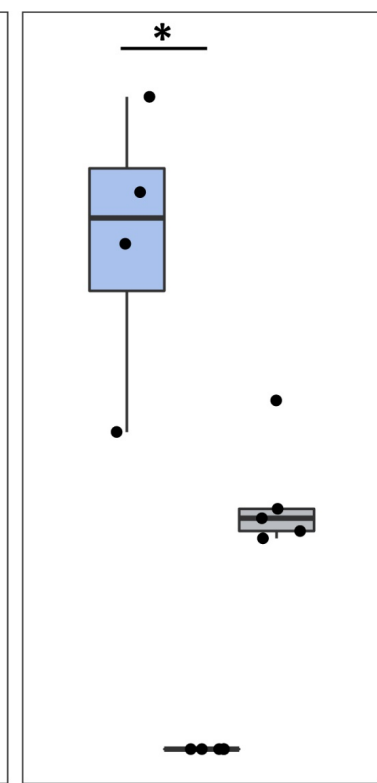

Sialidase

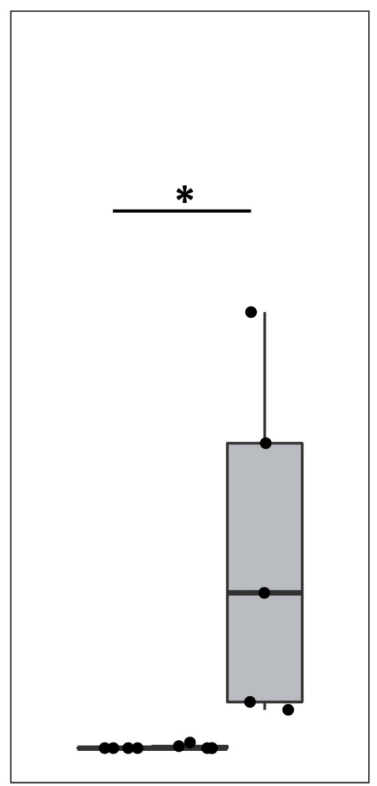

C5a peptidase 